\title{
Hiatal hernia on thoracic computed tomography in pulmonary fibrosis
}

\author{
Céline Tossier ${ }^{1}$, Clairelyne Dupin ${ }^{1}$, Laurent Plantier ${ }^{1,2,3}$, Julie Leger $^{4}$, \\ Thomas Flament ${ }^{1}$, Olivier Favelle ${ }^{5}$, Thierry Lecomte ${ }^{6}$, Patrice Diot ${ }^{1,2,3}$ and \\ Sylvain Marchand-Adam ${ }^{1,2,3}$
}

Affiliations: ${ }^{1}$ Service de Pneumologie, CHRU Tours, Tours, France. ${ }^{2}$ Université François Rabelais, UMR 1100, Tours, France. ${ }^{3}$ INSERM, Centre d'Etude des Pathologies Respiratoires, UMR 1100/EA6305, Tours, France. ${ }^{4} \mathrm{CHRU}$ Tours, INSERM, CIC 1415, Tours, France. ${ }^{5}$ Service de Radiologie, CHRU Tours, Tours, France. ${ }^{6}$ Service d'Hepato-gastro-enterologie Tours, CHRU Tours, Hôpital Bretonneau, Tours, France.

Correspondence: Sylvain Marchand-Adam, Service de Pneumologie et Explorations Fonctionnelles Respiratoires, Hôpital Bretonneau, 2 Boulevard Tonnellé, 37044, Tours Cedex, France.

E-mail: s.marchandadamauniv-tours.fr

ABSTRACT Gastro-oesophageal reflux has long been suspected of implication in the genesis and progression of idiopathic pulmonary fibrosis (IPF). We hypothesised that hiatal hernia may be more frequent in IPF than in other interstitial lung disease (ILD), and that hiatal hernia may be associated with more severe clinical characteristics in IPF.

We retrospectively compared the prevalence of hiatal hernia on computed tomographic (CT) scans in 79 patients with IPF and 103 patients with other ILD (17 scleroderma, 54 other connective tissue diseases and 32 chronic hypersensitivity pneumonitis). In the IPF group, we compared the clinical, biological, functional, CT scan characteristics and mortality of patients with hiatal hernia $(n=42)$ and without hiatal hernia $(n=37)$.

The prevalence of hiatal hernia on CT scan at IPF diagnosis was 53\%, similar to ILD associated with scleroderma, but significantly higher than in the two other ILD groups. The size of the hiatal hernia was not linked to either fibrosis CT scan scores, or reduction in lung function in any group. Mortality from respiratory causes was significantly higher among IPF patients with hiatal hernia than among those without hiatal hernia $(\mathrm{p}=0.009)$.

Hiatal hernia might have a specific role in IPF genesis, possibly due to pathological gastro-oesophageal reflux.

@ERSpublications

Hiatal hernia in idiopathic pulmonary fibrosis was associated with mortality from respiratory causes http://ow.ly/

This article has supplementary material available from erj.ersjournals.com

Received: Oct 302015 | Accepted after revision: April 032016 | First published online: May 122016

Conflict of interest: Disclosures can be found alongside this article at erj.ersjournals.com

Copyright OERS 2016 


\section{Introduction}

Idiopathic pulmonary fibrosis (IPF) is a rare and severe disease with median survival of $\sim 3$ years [1]. Several risk factors have been identified, such as age, genetic predisposition, tobacco exposure and gastro-oesophageal reflux (GOR) [2]. Prevalence of GOR in IPF is high and may affect $87 \%$ of patients, of whom $47 \%$ report symptoms $[2,3]$. In a retrospective study, treatment with proton pump inhibitors (PPIs) or surgical fundoplication appeared to stabilise lung function and decrease exacerbations in patients with IPF $[4,5]$. GOR has long been implicated in the genesis and progression of IPF through repeated microaspiration of gastric secretions, leading to recurrent lung tissue injury [6]. In support of this hypothesis, LeE et al. [7] observed high pepsin concentrations in the bronchoalveolar lavage fluid (BALF) of patients with IPF, and even higher concentrations during exacerbations of IPF. Recently, an association has been observed between the presence of antibodies against Helicobacter pylori in serum and increased severity of IPF [8]. The American Thoracic Society (ATS)/European Respiratory Society (ERS)/Japanese Respiratory Society (JRS)/ Latin American Thoracic Association (ALAT) 2011 guidelines for the management of IPF recommend treating GOR even if it is asymptomatic [1]. This recommendation is based upon "very low-quality evidence". The 2015 guideline update [9] suggests that clinicians use regular anti-acid treatment, and also stresses the need for additional research using randomised clinical trials. Hiatal hernia has been recognised as a major factor that predisposes individuals to develop GOR [10]. It may increase the duration of lower oesophageal sphincter relaxation and the risk of oesophagitis. In line with the high prevalence of GOR in IPF, hiatal hernia was detected using computed tomography (CT) in $39 \%$ of IPF patients versus $13.3 \%$ in chronic obstructive pulmonary disease (COPD) and $16.7 \%$ in asthma [11]. However, it remains to be determined whether the presence of a hiatal hernia has prognostic implications for patients with IPF, or whether hiatal hernia formation is merely the consequence of increased lung retraction forces (caused by lung fibrosis) pulling the oesophageal hiatus in the diaphragmatic muscle upward, with negligible impact on IPF pathogenesis and disease course.

To address this question, we retrospectively compared the prevalence of hiatal hernia on CT scans in IPF patients and patients with other interstitial lung disease (ILD) of similar severity. We tested two hypotheses: 1) that hiatal hernia is more frequent in IPF; and 2) that hiatal hernia is not linked to the severity of fibrosis. To assess whether hiatal hernia plays a role in the progression of IPF, we also investigated whether there was a difference between the survival of IPF patients with and without hiatal hernia.

\section{Materials and methods}

\section{Patients}

Criteria for inclusion were chronic ILD, defined by persistent ILD at CT scan over $\geqslant 6$ months, and the availability of pulmonary function tests performed within 6 months of the initial clinic visit. The files of patients with pulmonary fibrosis $(n=352)$ attending the respiratory medicine department of Tours University (Tours, France) between October 2006 and May 2013 were reviewed (fig. 1). We excluded 170 patients, either because of the absence of a lung CT scan $(n=46)$ or because samples were too small (sarcoidosis $n=2$; silicosis $n=1$; asbestosis $n=10$; and idiopathic nonspecific interstitial pneumonia $n=11$ ), or because of a diagnosis of drug-induced interstitial lung disease $(n=34)$. The other 66 patients were excluded because ILD was unclassifiable (defined as patients without a specific ILD diagnosis following multidisciplinary review of clinical, radiological and pathological data). Study subjects included patients with IPF, ILD associated with connective tissue disease (CTD-ILD) and chronic hypersensitivity pneumonitis (CHP), in accordance with

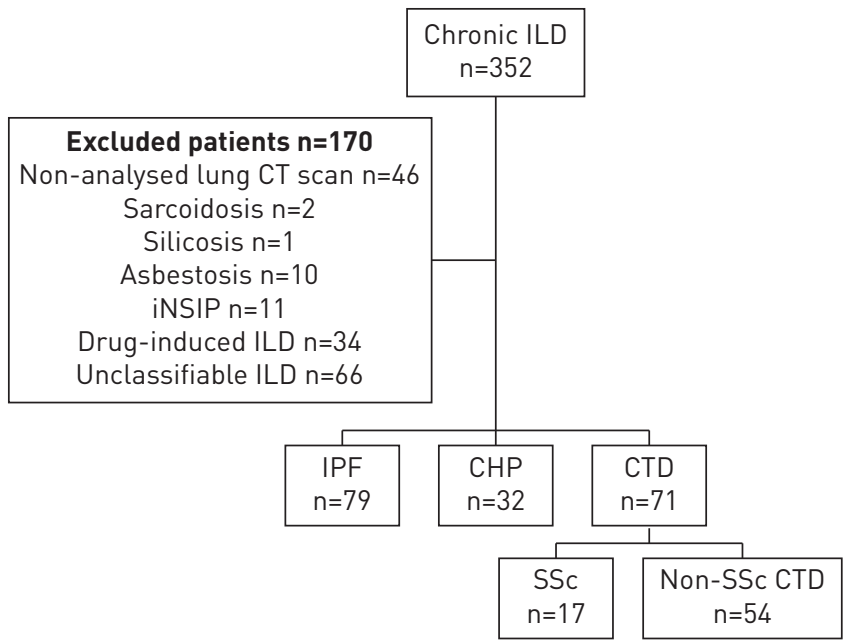

FIGURE 1 Trial profile of interstitial lung disease (ILD) between October 2006 and May 2013 at the University Hospital of Tours (Tours, France). CT: computed tomography; iNSIP: idiopathic nonspecific interstitial pneumonia; IPF: idiopathic pulmonary fibrosis; CHP: chronic hypersensitivity pneumonitis; CTD: Connective tissue disease; SSc: scleroderma. 
established criteria. The diagnosis of IPF was based on ATS/ERS/JRS/ALAT criteria [1]. The diagnosis of CHP was based on the criteria established by LACASSE et al. [12]. CTD with lung involvement was identified on the basis of criteria described in the literature [13-15].

\section{Ethics statement}

French legislation does not require the informed consent of patients for the retrospective collection of data that conform to current practice. However, the study protocol was evaluated and approved by the institutional review board of the French Society for Respiratory Medicine (Société de Pneumologie de Langue Française) (CEPRO \#2012-015). The database was anonymous and complied with the restrictive requirements of the Commission Nationale de l'Informatique et des Libertés, which ensures the application of data privacy laws in France.

\section{Data collection}

Data were collected from the retrospective review of electronic medical records. We collected clinical data (age at the time of diagnosis, sex, body mass index, smoking habits, presence or absence of GOR symptoms including heartburn, treatment with PPI, oxygen therapy and treatment with immunosuppressant drugs), respiratory symptoms (cough or dyspnoea as classified by the modified Medical Research Council scale), pulmonary hypertension (systolic pulmonary arterial pressure $>40 \mathrm{mmHg}$ as assessed by echocardiography), biological parameters (lactate dehydrogenase, D-dimers, brain natriuretic peptide and blood neutrophils), pulmonary function data expressed as a percentage of the predicted value, arterial blood gases, BALF cell count and the occurrence of acute exacerbations [16]. In addition, for IPF patients, we collected data of mortality between diagnosis and the end of the study. Mortality from respiratory causes is defined as the occurrence of death at the time of an exacerbation or end stage of IPF.

\section{Pulmonary function tests}

Lung function tests were performed using Sensormedics Vmax Encore plethysmography (Carefusion, San Diego, CA, USA) in accordance with ATS/ERS recommendations [17] and European Community for Steel and Coal reference equations [18]. Forced vital capacity (FVC), total lung capacity and forced expiratory volume in $1 \mathrm{~s}$ values were expressed as percentages of predicted values according to sex, height and age.

\section{Thoracic CT}

Thoracic CT scans of the 182 patients were analysed retrospectively. Thoracic CT scans were performed using a 64-slice CT Scanner (Philips Healthcare, DA Best, the Netherlands). They were analysed simultaneously by a blinded chest radiologist (O. Favelle) and lung specialist (S. Marchand-Adam), who reached a clear consensus about the quantification of lesions. Observers were blinded to the patients' clinical and functional details, in accordance with previously published criteria [19].

In brief, lung abnormalities were evaluated for their presence, number, extent and distribution, as described by Sumikawa et al. [20]. Opacities including ground-glass opacities, micronodules and alveolar consolidations were considered active alveolar lesions and quantified as an alveolitis score; opacities such as linear opacities, traction bronchiectasis, lobular distortions, bulla formations, cysts and honeycombing were considered fibrotic lesion expressions and quantified as a fibrosis lesion score. These two patterns were scored separately in six areas of the lung, defined as: upper zones located above the level of the carina; middle zones located between the level of the carina and the level of the inferior pulmonary veins; and lower zones located below the level of the inferior pulmonary veins. The intensity of alveolar opacities on thin-section high-resolution CT was scored semi-quantitatively in each area, from 0 (absent) to 4 (present in $<25 \%, 25-50 \%, 50-75 \%$ and $>75 \%$ of lung parenchyma in the area). The intensity of fibrotic opacities was quantified by the same process, from 0 (absent) to 4 (present in $<5 \%, 5-25 \%, 25-50 \%$ and $>50 \%)$ in each area. The total alveolar and fibrotic scores thus ranged between 0 and 24 . Using the same method, we also recorded lobular attenuations and reticulations.

In addition, CT scans were evaluated for the presence or absence of hiatal hernia. If present, hiatal hernia was classified as type I-IV, as previously described (fig. 2) [21]. Agreement between the radiologist and lung specialist was good $(\kappa=0.715,95 \%$ CI $0.621-0.793)$. The measurements of the frontal and sagittal diameters $(\mathrm{mm})$ of the oesophagus in its lower portion in the mediastinum (inferior third) on axial sections enabled us to calculate the oesophageal or intrathoracic stomach area. The presence of air or the presence of food/fluid/air-fluid level in the oesophagus was recorded.

\section{Statistical analysis}

A nonparametric method was used, and all data are presented as median (range). Continuous data were compared between ILD groups using the Kruskal-Wallis test. The Mann-Whitney U-test was performed to compare the groups of IPF patients with type I hiatal hernia, type II, III or IV hiatal hernia and without 

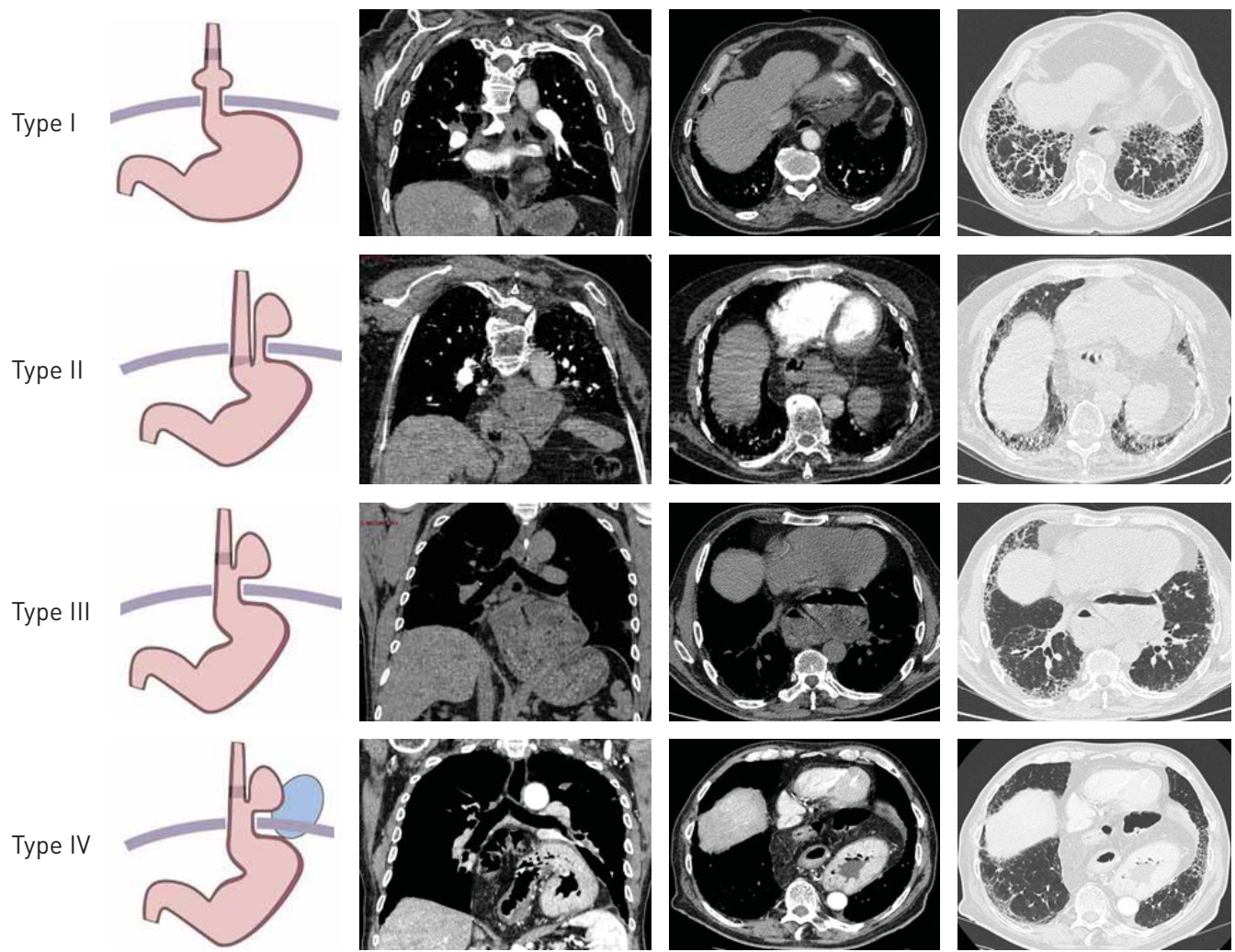

FIGURE 2 Representative computed tomographic images of idiopathic pulmonary fibrosis patients with type I, II, III and IV hiatal hernia. Type I: migration of oesophagogastric junction through the gap in the cephalad direction. Type II: the oesophagogastric junction remains in normal position under diaphragm; migration of gastric fundus in chest. Type III: migration of oesophagogastric junction in chest, along with fundus and other portions of stomach. Type IV: migration of other structures and organs in chest.

hiatal hernia. Chi-squared and Fisher tests were used to compare categorical data for each group. Correlations were assessed using Spearman's rank order test.

We studied the occurrence of death from respiratory causes in the IPF groups using a survival model with competing risks. The times considered in this analysis were from the date of diagnosis up to the date of death, in months. GRAY's [22] test compares the cumulative incidence curves by group. We estimated the hazard ratio (HR) associated with FINE and GRAY's [23] multistate model for competing risks, its 95\% confidence interval and the p-value. First, a univariate analysis was performed, taking the presence of hiatal hernia as the only explicative variable. Then, we used a multivariate model with age, vital capacity and fibrosis score as covariables in addition to the presence of hiatal hernia. The cumulative incidence curves for respiratory causes of death were plotted for each group (with and without hiatal hernia). Statistical significance was defined as $\mathrm{p}<0.05$.

\section{Results}

Clinical, functional and CT scan data of patients with IPF and other ILD

Patients with ILD were divided into four groups: IPF ( $n=79)$, CHP $(n=32)$, lung involvement caused by scleroderma (SSc; $\mathrm{n}=17$ ) and other CTDs excluding scleroderma (non-SSc CTD; $\mathrm{n}=54$, including 17 rheumatoid arthritis, 12 autoimmune myositis, 11 Sjögren's syndrome, three microscopic polyangiitis and 11 interstitial pneumonia with autoimmune features (IPAF)). The IPF diagnostic criteria were based on a definite pattern of usual interstitial pneumonia (UIP) on CT scan ( $\mathrm{n}=71)$ or a combination of a possible CT UIP pattern and a definite histopathological UIP pattern shown by surgical lung biopsy $(\mathrm{n}=8)$ (online supplementary table S3). Scleroderma was treated separately from other CTDs because it is frequently accompanied by specific oesophageal injuries. One patient with IPAF had specific oesophageal injuries.

Clinical and functional data are presented in table 1. Patients with IPF were older than those with SSc $(\mathrm{p}=0.001)$. There was no difference in the age of patients with IPF and other ILD. There were smaller proportions of women and more smokers in the IPF group than in the CHP, SSc and non-SSc CTD groups $(\mathrm{p}<0.05)$. There was no significant difference in respiratory symptoms. Frequency of acute exacerbations was identical in the IPF and CHP patients, but this was higher than in all the other groups 


\begin{tabular}{|c|c|c|c|c|c|}
\hline & IPF & CHP & SSc & Non-SSc CTD & p-value \\
\hline Subjects & 79 & 32 & 17 & 54 & \\
\hline Age at diagnosis of ILD years & $73(53-89)$ & $70(41-90)$ & $57(39-80)$ & $68(28-86)$ & 0.001 \\
\hline Male & $65 / 79(82)$ & $17 / 32(53)$ & $2 / 17(12)$ & $27 / 54(50)$ & 0.001 \\
\hline $\mathrm{BMI}^{\#} \mathrm{~kg} \cdot \mathrm{m}^{-2}$ & $27(18-37)$ & $24(16-47)$ & $23(16-32)$ & $28(18-37)$ & 0.093 \\
\hline \multicolumn{6}{|l|}{ Smoking history } \\
\hline Current smokers & $6 / 77(8)$ & $0 / 32(0)$ & $1 / 14(7)$ & $2 / 54(4)$ & 0.001 \\
\hline Ex-smokers & $54 / 77(70)$ & $12 / 32(38)$ & $2 / 14(14)$ & $22 / 54(41)$ & \\
\hline Never-smokers & $17 / 77(22)$ & $20 / 32(63)$ & $11 / 14(79)$ & $30 / 54(56)$ & \\
\hline Pack-years & $20(0-120)$ & $0(0-45)$ & $0(0-20)$ & $0(0-80)$ & 0.001 \\
\hline Cough & 45/77 (58) & $20 / 30(67)$ & $11 / 16$ (69) & $28 / 54$ (52) & 0.475 \\
\hline Dyspnoea mMRC score & & & & & 0.390 \\
\hline Grade I & $13 / 74(18)$ & $2 / 30(7)$ & $1 / 16(6)$ & $6 / 52(12)$ & \\
\hline Grade II & $24 / 74(32)$ & $10 / 30(33)$ & $7 / 16(44)$ & $26 / 52(50)$ & \\
\hline Grade III & $22 / 74(30)$ & $10 / 30$ (33) & $6 / 16$ (38) & $14 / 52$ (27) & \\
\hline Grade IV & $15 / 74(20)$ & $8 / 30(27)$ & $2 / 16$ (13) & $6 / 52(12)$ & \\
\hline Exacerbations & 42/79 (53) & $15 / 32$ (47) & $5 / 17$ (29) & $12 / 54$ (22) & 0.003 \\
\hline FVC $\%$ pred & $71(38-141)$ & $59(35-126)$ & $87(29-160)$ & $79(42-125)$ & 0.028 \\
\hline TLC ${ }^{\pi} \%$ pred & $66(42-101)$ & $60(40-112)$ & $84(26-105)$ & $72(52-103)$ & 0.058 \\
\hline FEV $_{1} / F^{\text {Iी }} \%$ & $83(46-115)$ & $84(74-100)$ & 82 (49-94) & $81(55-138)$ & 0.500 \\
\hline FEV ${ }^{\pi} \%$ pred & 78 (39-148) & $66(36-124)$ & $69(26-161)$ & $79(47-129)$ & 0.111 \\
\hline Duco" \% pred & $44(14-78)$ & $39(17-88)$ & $34(13-68)$ & $44(16-82)$ & 0.264 \\
\hline $\mathrm{PaO}_{2}^{+} \mathrm{mmHg}$ & $73(36-110)$ & $72(46-95)$ & $73(62-80)$ & 77 (53-102) & 0.270 \\
\hline $\mathrm{PaCO}_{2}^{+} \mathrm{mmHg}$ & $39(27-75)$ & $40(32-65)$ & $38(31-49)$ & $38(30-47)$ & 0.140 \\
\hline Alveolitis score & $8(1-23)$ & $12(0-23)$ & $10(2-21)$ & $10(0-23)$ & 0.009 \\
\hline Fibrosis score & $13(3-24)$ & $12(2-22)$ & $9(2-16)$ & $11(2-24)$ & 0.012 \\
\hline $\begin{array}{l}\text { Lobular areas of decreased } \\
\text { attenuation }\end{array}$ & $2(0-6)$ & $3(0-6)$ & $0(0-5)$ & $1(0-6)$ & 0.001 \\
\hline Thickening of interlobular septa & $3(0-6)$ & $2(0-6)$ & $0(0-3)$ & $2(0-6)$ & 0.100 \\
\hline Consolidation & $0(0-0)$ & $0(0-5)$ & $1(0-5)$ & $0(0-6)$ & 0.004 \\
\hline
\end{tabular}

Data are presented as $\mathrm{n}$, median (range) or $\mathrm{n} / \mathrm{N}(\%)$, unless otherwise stated. IPF: idiopathic pulmonary fibrosis; CHP: chronic hypersensitivity pneumonitis; SSc: scleroderma; CTD: connective tissue disease; BMI: body mass index; mMRC: modified Medical Research Council; FVC: forced vital capacity; TLC: total lung capacity; FEV1: forced expiratory volume in $1 \mathrm{~s} ; D L C O$ : diffusing capacity of the lung for carbon monoxide; $\mathrm{PaO}_{2}$ : arterial oxygen tension; $\mathrm{PaCO}_{2}$ : arterial carbon dioxide tension. ${ }^{\#}$ : data available for 69 IPF, $28 \mathrm{CHP}, 15 \mathrm{SSc}$ and 50 non-SSc CTD patients; ": data available for 71 IPF, 23 CHP, 16 SSc and 47 non-SSc CTD patients; ${ }^{+}$: data available for 70 IPF, 26 CHP, nine SSc and nine non-SSc CTD patients.

$(\mathrm{p}=0.01)$. FVC in IPF patients was higher than in CHP patients $(\mathrm{p}=0.01)$ but similar to other ILD groups. BALF cell counts are shown in online supplementary table S1. Total fibrosis scores were higher in patients with IPF than in those with SSc $(\mathrm{p}=0.03)$, but were similar to the other groups.

Comparison of GOR symptoms and hiatal hernia of IPF patients with those of other ILD patients The IPF and SSc patients showed no difference in GOR symptoms (fig. 3a), PPI medication, hiatal hernia characteristics (prevalence, oesophageal or intrathoracic stomach area and presence of food/fluid/air-fluid level in the oesophagus) (table 2).

GOR symptoms, medication with PPI and the prevalence of hiatal hernia were more frequent in IPF than in CHP $(p<0.05)$ and non-SSc CTD $(p<0.05)$ (fig. 3a and table 2$)$. The prevalence of stages II, III or IV hiatal hernia was significantly higher in IPF patients than in non-SSc CTD patients $(p=0.027)$. The oesophageal or intrathoracic stomach area in the inferior third of the mediastinum was greater in the IPF group than in non-SSc CTD patients $(\mathrm{p}=0.003)$.

\section{Comparison of IPF patients with and without hiatal hernia}

IPF patients with type I hiatal hernia $(n=31)$ or with type II, III or IV hiatal hernia $(n=11)$ were older than those without hiatal hernia ( $\mathrm{p}=0.01$ and $\mathrm{p}=0.001$, respectively) and reported more GOR symptoms $(58 \%$ versus $31 \%, \mathrm{p}=0.034$ ) (table 3 ). GOR symptoms were significantly more frequent in IPF patients with type 

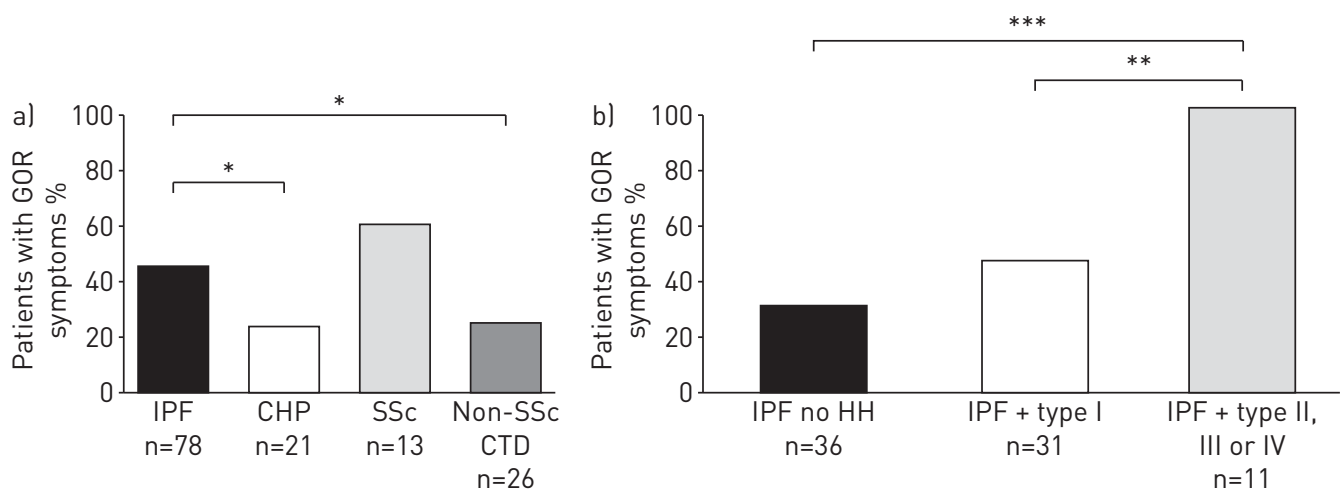

FIGURE 3 Frequency of gastro-oesophageal (GOR) symptoms in a) four groups at diagnosis of interstitial lung disease (idiopathic pulmonary fibrosis (IPF), chronic hypersensitivity pneumonitis (CHP), scleroderma (SSc), and non-SSc connective tissue disease (CTD)) and b) IPF patients without hiatal hernia (HH), with type I HH and with type II, III or IV HH. IPF patients have significantly more GOR symptoms than CHP and non-SSc CTD patients. IPF patients with type II, III or IV HH have more GOR symptoms than IPF patients with type I HH or IPF patients without $\mathrm{HH} .{ }^{*}: \mathrm{p}<0.05{ }^{* *}: \mathrm{p}<0.01 ;^{* * *}: \mathrm{p}<0.001$.

II, III or IV hiatal hernia than in the other two groups (fig. 3b). BALF cell counts, treatment with PPI, tobacco smoking, cough, obesity and exacerbation rates did not differ between IPF patients with and without hiatal hernia. CT fibrosis scores and respiratory function did not differ between IPF patients with and without hiatal hernia.

Factors predictive of mortality

Median survival was 31 months in the 42 IPF patients with hiatal hernia, and 40 months in the 37 IPF patients without hiatal hernia. In the IPF group with hiatal hernia, 26 (62\%) patients died, 23 from respiratory causes, two from cardiovascular diseases and one from lung cancer, while in the IPF group without hiatal hernia, 21 (57\%) patients died: 14 from respiratory causes, three from cardiovascular diseases, three from lung cancer and one from sepsis. The time between diagnosis and death from respiratory causes was lower in IPF patients with hiatal hernia than without hiatal hernia (Gray's test: $p=0.009$ ) (fig. 4). As shown in table 4, multivariate analysis with competing risks identified two independent predictors of mortality from respiratory causes in IPF patients: presence of hiatal hernia (HR 2.41, 95\% CI 1.09-5.35; $\mathrm{p}=0.030$ ) and higher CT fibrosis score (HR 1.13, 95\% CI 1.03-1.24; $\mathrm{p}=0.011$ ). The results were identical, taking into account only patients receiving PPI treatment (online supplementary table S2). Two IPF patients with hiatal hernia had fundoplication and died 29 and 66 months after IPF diagnosis.

\section{Discussion}

The main results of our study are 1) the increased prevalence of hiatal hernia in IPF compared to the other ILDs studied and 2) the association of increased mortality risk from respiratory causes with the presence of a hiatal hernia.

We investigated the presence or absence of hiatal hernia in the inferior third of the posterior mediastinum using thoracic CT scans. The diagnosis of hiatal hernia is classically obtained by oesophageal transit of a contrast agent or by oesophageal endoscopy, but thoracic and abdominal CT scanning is also considered a

TABLE 2 Gastro-oesophageal reflux (GOR) symptoms and hiatal hernia at diagnosis of interstitial lung disease of the 182 patients

\begin{tabular}{|c|c|c|c|c|c|}
\hline & IPF & CHP & SSc & Non-SSc CTD & p-value \\
\hline Subjects & 79 & 32 & 17 & 54 & \\
\hline GOR symptoms & $36 / 78(46)$ & $5 / 22(23)$ & $8 / 13(62)$ & $9 / 35(26)$ & 0.034 \\
\hline Patients treated with a PPI & $57 / 77(74)$ & $18 / 31(58)$ & $15 / 17(88)$ & $26 / 52(50)$ & 0.029 \\
\hline Presence of hiatal hernia on CT scan & $42 / 79$ (53) & $9 / 32(28)$ & $10 / 17(59)$ & $18 / 54(33)$ & 0.017 \\
\hline Presence of type II, III or IV hiatal hernia & $11 / 79(14)$ & $1 / 32(3)$ & $1 / 17(6)$ & $1 / 54(2)$ & 0.005 \\
\hline Oesophageal or intra-thoracic stomach area $\mathrm{mm}^{2}$ & $339(75-6406)$ & $254(94-659)$ & $505(150-1648)$ & $243(102-650)$ & 0.014 \\
\hline Presence of air in the oesophagus & $72 / 79(91)$ & $27 / 32(84)$ & $17 / 17(100)$ & $51 / 54(94)$ & 0.229 \\
\hline Presence of food/fluid/air-fluid level in the oesophagus & $7 / 79(9)$ & $2 / 32(6)$ & $5 / 17(29)$ & $1 / 54(2)$ & 0.005 \\
\hline
\end{tabular}

Data are presented as $\mathrm{n}, \mathrm{n} / \mathrm{N}(\%)$ or median (range), unless otherwise stated. IPF: idiopathic pulmonary fibrosis; CHP: chronic hypersensitivity pneumonitis; SSc: scleroderma; CTD: connective tissue disease; PPI: proton pump inhibitor; CT: computed tomography. 
TABLE 3 Demographic and clinical characteristics at diagnosis of idiopathic pulmonary fibrosis (IPF) of 79 patients, according to type of hiatal hernia

IPF patients without hiatal hernia

\section{IPF patients with \\ type I hiatal hernia}

IPF patients with type II, III or IV hiatal hernia p-value

31

$74(66-89)$

$24 / 31(77)$

29 (19-37)

33/37 (89)

$27(18-32)$

$4 / 36(11)$

$27 / 36(75)$

$5 / 36(14)$

$30(0-105)$

$21 / 35(60)$

$11 / 36(31)$

$27 / 36$ (75)

19/37 (51)

$69(40-141)$

64 (47-99)

85 (46-115)

80 (52-148)

42 (16-78)

71 (43-98)

38 (29-57)

$9(1-18)$

13 (4-19)

$11 / 37$ (30)

$2(0-5)$

2 (0-6)
$1 / 30$ (3)
$22 / 30(73)$
7 (23)
$20(0-65)$
$18 / 31$ (58)
$14 / 31$ (45)
$19 / 30(63)$
$18 / 31$ (58)
75 (38-132)
68 (44-101)
82 (62-108)
81 (39-125)
$42(28-68)$
$73(36-106)$
$38(30-75)$
8 (2-23)
12 (3-24)
$9 / 31$ (29)
$2(0-6)$
$3(0-6)$

11

$78(61-86)$
$8 / 11(73)$

25 (20. 29)

$1 / 11$ (9)

$5 / 11$ (45)

$5 / 11$ (45)

15(0-120)

6/11 (54)

$11 / 11$ (100)

$11 / 11$ (100)

5/11 (45)

74 (51-110)

62 (42-82)

85 (79-108)

77 (62-115)

$46(14-60)$

76 (39-110)

$39(27-54)$

$6(4-9)$

14 (7-18)

$4 / 11$ (36)

$1(0-2)$

$3(0-6)$
0.008

0.294

0.080

0.194

0.255

0.981

0.001

0.059

0.737

0.801

0.427

0.440

0.877

0.900

0.370

0.814

0.155

0.334

1.000

0.061

0.125

Data are presented as $\mathrm{n}$, median (range) or $\mathrm{n} / \mathrm{N}(\%)$, unless otherwise stated. ILD: interstitial lung disease; BMI: body mass index; GOR: gastro-oesophageal reflux; PPI: proton pump inhibitor; FVC: forced vital capacity; TLC: total lung capacity; FEV1: forced expiratory volume in $1 \mathrm{~s}$; $D\left\llcorner C 0\right.$ : diffusing capacity of the lung for carbon monoxide; $\mathrm{PaO}_{2}$ : arterial oxygen tension; $\mathrm{PaCO}_{2}$ : arterial carbon dioxide tension. ${ }^{\#}$ : data available for 30 IPF patients without hiatal hernia, 28 IPF patients with type I hiatal hernia, and 11 IPF patients with type II, III or IV hiatal hernia; ": data available for 33 IPF patients without hiatal hernia, 27 IPF patients with type I hiatal hernia, and 11 IPF patients with type II, III or IV hiatal hernia: ${ }^{+}$: data available for 32 IPF patients without hiatal hernia, 21 IPF patients with type I hiatal hernia, and 9 IPF patients with type II, III or IV hiatal hernia.

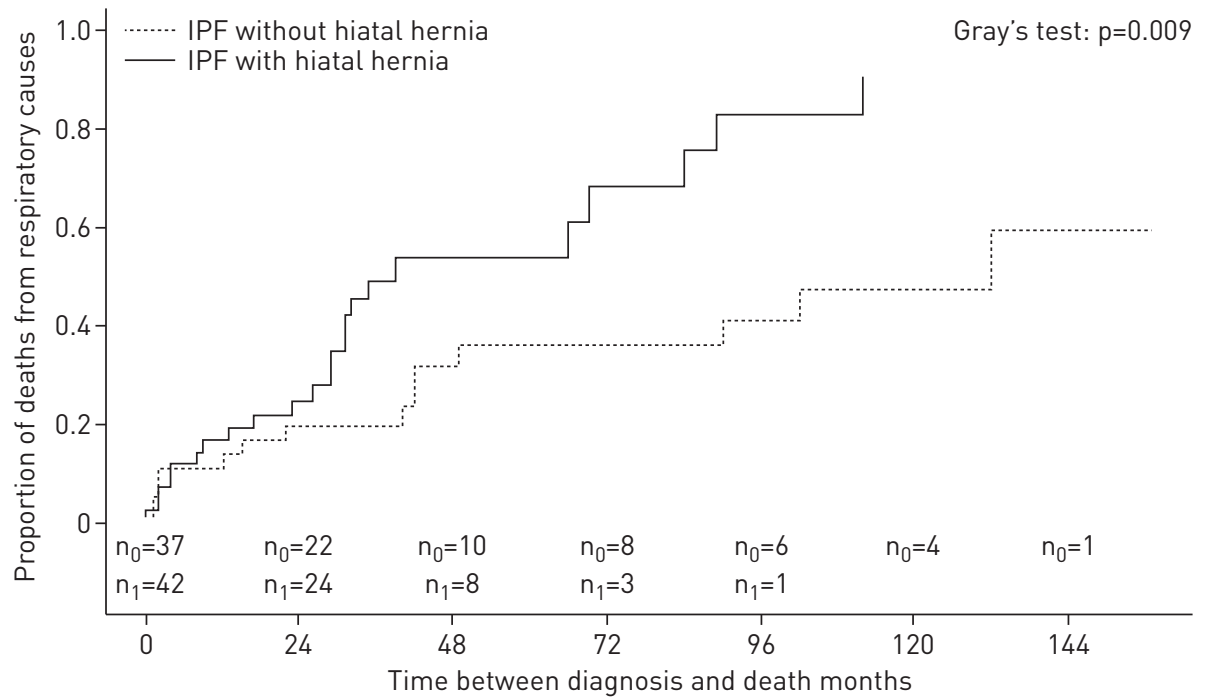

FIGURE 4 Comparison of mortality due to respiratory causes in idiopathic pulmonary fibrosis (IPF) patients with and without hiatal hernia $\left(\mathrm{n}_{1}\right.$ and $\mathrm{n}_{0}$, respectively). 
TABLE 4 Predictors of mortality from respiratory causes in patients with idiopathic pulmonary fibrosis (IPF), identified using a multivariate survival model with competing risks

\begin{tabular}{lcc} 
& Death from respiratory causes HR (95\% CI) & p-value \\
\hline Hiatal hernia & $2.43(1.09-5.43)$ & 0.030 \\
Age & $1.03(0.99-1.07)$ & 0.130 \\
FVC \% predicted & $0.99(0.98-1.01)$ & 0.450 \\
Fibrosis CT score & $1.13(1.03-1.24)$ & 0.011 \\
Proton pump inhibitor treatment & $1.15(0.43-3.09)$ & 0.870 \\
\hline
\end{tabular}

n=70. FVC: forced vital capacity; CT: computed tomography.

reliable tool for the diagnosis and evaluation of hiatal hernia [24-26]. The hiatal hernia can be classified by reconstructing CT images in the coronal plane (fig. 2) [25]. Reconstructions of the CT images enabled us to calculate the maximal area of the oesophagus and stomach from measurements of the sagittal and coronal diameters in the inferior third of the mediastinum. This quantitative method was used by Vonk et al. [27], who reported a dilatation of the oesophagus in $62 \%$ of patients with SSc from measurement of the oesophageal diameter in the coronal plane, versus $12 \%$ in controls. In our study, hiatal hernia was present in $59 \%$ of patients with SSc, suggesting that CT scanning is indeed a robust method for diagnosis of hiatal hernia.

In our study, hiatal hernia was present in 53\% of IPF patients. This result is consistent with those of LEE et al. [7], whereas Nотн et al. [11] observed a frequency of hiatal hernia of $39 \%$ in IPF, compared with $13 \%$ in COPD and $17 \%$ in asthma, which is close to the prevalence of hiatal hernia in the general population (17\%) [28]. This difference could reflect different ways of interpreting CT scans. In addition to the known increased frequency of hiatal hernia in IPF, our data show the higher frequency of more severe hiatal hernia (type II, III or IV) in IPF than in other ILD (14\% versus 2-6\%). Hiatal hernia is recognised as an independent predictive factor of reflux [29]. Accordingly, our IPF patients with hiatal hernia (in particular type II, III or IV hiatal hernia) reported more GOR symptoms than patients without hiatal hernia.

The higher frequency of hiatal hernia in IPF raises the question of whether hiatal hernia is a comorbidity, a consequence or a driver of IPF. Risk factors of hiatal hernia include increases in intra-abdominal pressure as observed in obesity, pregnancy, chronic ascites, abdominal trauma, chronic cough, congenital abnormalities (short oesophagus) and tobacco smoking [28]. In patients with IPF, cough, obesity and tobacco smoking did not differ between the groups with and without hiatal hernia. The occurrence of hiatal hernia increases with age; its prevalence is estimated to be $\sim 10 \%$ in people aged $<40$ years, and $56 \%$ in people aged $>70$ years [30]. In our study, IPF patients with hiatal hernia were older than those without hiatal hernia. However, except for ILD associated with SSc, the age of patients with IPF was not significantly different from that of patients with other ILDs (median 74 years), while hiatal hernia was present in $53 \%$ of patients with IPF compared to $28 \%$ of CHP patients or $33 \%$ of non-SSc CTD patients. Consequently, we believe that the older age of patients with IPF is probably not the key explanation for the higher frequency of hiatal hernia in this disease.

One possibility for the higher prevalence of hiatal hernia in IPF is that the reduction in lung compliance secondary to extensive fibrosis may lead to a lateral shift of diaphragmatic pillars, followed by the stomach rising into the thorax. However, we did not observe any difference in CT lung fibrosis scores or FVC, which indirectly reflects lung compliance, between patients with IPF and patients with CHP, whereas hiatal hernia frequency was higher in IPF than in the latter group. Likewise, CT fibrosis scores and respiratory function did not differ between IPF patients with and without hiatal hernia. Thus, although we did not measure intrathoracic pressure in our patients, these results suggest that retraction forces exerted by fibrotic lungs do not play a predominant role in the increased frequency of hiatal hernia in IPF.

Conversely, hiatal hernia may play a role in the pathogenesis of IPF by increasing the likelihood of GOR and thus the risk of microaspirations in the lung [31]. SAVARINO et al. found a good correlation between degree of reflux and CT scan fibrosis scores for scleroderma [32] and IPF [33]. The role of microaspiration in the genesis or progression of IPF is supported by LEE et al. [7] who reported measurable concentrations of pepsin in the BALF of IPF patients, both at steady state and during exacerbations, which suggests that microaspirations of gastric fluid are frequent in IPF; and by data extracted from the placebo arms of clinical trials where IPF patients treated with anti-acids had a slower decline in FVC compared to patients not receiving these drugs [5].

A major finding of our study is that the presence of hiatal hernia was a predictor of mortality from respiratory causes in IPF patients. In contrast, Nотн et al. [11] did not observe any link between the presence of hiatal hernia and severity of IPF, except in IPF patients with hiatal hernia and reflux treated by PPI, who have better lung function than patients with IPF, hiatal hernia and untreated reflux. In our study, 
we do not know when antireflux therapy was initiated for patients already on therapy before IPF diagnosis, or if it was initiated later for those not on therapy at entry. There were no controls for treatment, nor do we have any outcome data. This limits the interpretation of results regarding the role of PPI therapy in IPF care. Since hiatal hernia is associated with an increased risk of recurrence and failure of medical therapy, including PPI [25], it would be interesting to evaluate surgical correction of hiatal hernia in these patients.

We recognise that this study has limitations. First, our study was observational, with data gathered retrospectively. Second, our samples were small, especially in the IPF group with type II, III or IV hiatal hernia. This small sample size limits any firm conclusions that can be drawn from the data. Third, the study was conducted before pirfenidone and nintedanib became available. The effect of antifibrotic therapy in IPF patients with hiatal hernia is not known. Finally, we did not use pH-metry to investigate the presence of GOR. We cannot affirm that hiatal hernia is always accompanied by GOR, even in patients who show GOR symptoms.

To conclude, we found a higher prevalence of hiatal hernia in patients with IPF than in those with other ILD, and a similar severity of lung fibrosis. The presence of hiatal hernia was associated with a higher risk of mortality from respiratory causes. Our results support the hypothesis that dysfunction of the upper digestive tract could play a specific role in the physiopathology of IPF.

\section{References}

1 Raghu G, Collard HR, Egan JJ, et al. An official ATS/ERS/JRS/ALAT statement: idiopathic pulmonary fibrosis: evidence-based guidelines for diagnosis and management. Am J Respir Crit Care Med 2011; 183: 788-824.

2 Fahim A, Crooks M, Hart SP. Gastroesophageal reflux and idiopathic pulmonary fibrosis: a review. Pulm Med 2011; 2011: 634613 .

3 Raghu G, Freudenberger TD, Yang S, et al. High prevalence of abnormal acid gastro-oesophageal reflux in idiopathic pulmonary fibrosis. Eur Respir J 2006; 27: 136-142.

4 Raghu G, Yang ST, Spada C, et al. Sole treatment of acid gastroesophageal reflux in idiopathic pulmonary fibrosis: a case series. Chest 2006; 129: 794-800.

5 Lee JS, Ryu JH, Elicker BM, et al. Gastroesophageal reflux therapy is associated with longer survival in patients with idiopathic pulmonary fibrosis. Am J Respir Crit Care Med 2011; 184: 1390-1394.

6 Bandeira CD, Rubin AS, Cardoso PFG, et al. Prevalence of gastroesophageal reflux disease in patients with idiopathic pulmonary fibrosis. J Bras Pneumol 2009; 35: 1182-1189.

7 Lee JS, Song JW, Wolters PJ, et al. Bronchoalveolar lavage pepsin in acute exacerbation of idiopathic pulmonary fibrosis. Eur Respir J 2012; 39: 352-358.

8 Bennett D, Bargagli E, Refini RM, et al. Helicobacter pylori seroprevalence in patients with idiopathic pulmonary fibrosis. Eur Respir J 2014; 43: 635-638.

9 Raghu G, Rochwerg B, Zhang Y, et al. An official ATS/ERS/JRS/ALAT clinical practice guideline: treatment of idiopathic pulmonary fibrosis. An update of the 2011 clinical practice guideline. Am J Respir Crit Care Med 2015; 192: e3-e19.

10 Yeom JS, Park HJ, Cho JS, et al. Reflux esophagitis and its relationship to hiatal hernia. J Korean Med Sci 1999; 14: 253-256.

11 Noth I, Zangan SM, Soares RV, et al. Prevalence of hiatal hernia by blinded multidetector CT in patients with idiopathic pulmonary fibrosis. Eur Respir J 2012; 39: 344-351.

12 Lacasse Y, Selman M, Costabel U, et al. Classification of hypersensitivity pneumonitis: a hypothesis. Int Arch Allergy Immunol 2009; 149: 161-166.

13 Vitali C, Bombardieri S, Jonsson R, et al. Classification criteria for Sjögren's syndrome: a revised version of the European criteria proposed by the American-European Consensus Group. Ann Rheum Dis 2002; 61: 554-558.

14 Kinder BW, Collard HR, Koth L, et al. Idiopathic nonspecific interstitial pneumonia: lung manifestation of undifferentiated connective tissue disease? Am J Respir Crit Care Med 2007; 176: 691-697.

15 Arnett FC, Edworthy SM, Bloch DA, et al. The American Rheumatism Association 1987 revised criteria for the classification of rheumatoid arthritis. Arthritis Rheum 1988; 31: 315-324.

16 Collard HR, Moore BB, Flaherty KR, et al. Acute exacerbations of idiopathic pulmonary fibrosis. Am J Respir Crit Care Med 2007; 176: 636-643

17 Laszlo G. Standardisation of lung function testing: helpful guidance from the ATS/ERS Task Force. Thorax 2006; 61: 744-746.

18 Quanjer PH, Tammeling GJ, Cotes JE, et al. Lung volumes and forced ventilatory flows. Report Working Party Standardization of Lung Function Tests, European Community for Steel and Coal. Official statement of the European Respiratory Society. Eur Respir J 1993; 16: Suppl. 16, 5-40.

19 Silva CIS, Müller NL, Lynch DA, et al. Chronic hypersensitivity pneumonitis: differentiation from idiopathic pulmonary fibrosis and nonspecific interstitial pneumonia by using thin-section CT. Radiology 2008; 246: 288-297.

20 Sumikawa H, Johkoh T, Fujimoto K, et al. Pathologically proved nonspecific interstitial pneumonia: CT pattern analysis as compared with usual interstitial pneumonia CT pattern. Radiology 2014; 272: 549-556.

21 Skinner DB. Hernias (hiatal, traumatic, and congenital). In: Berk JE, ed. Gastroenterology. 4th Edn. Philadelphia, WB Saunders, 1985; pp. 705-716.

22 Gray RJ. A class of K-sample tests for comparing the cumulative incidence of a competing risk. Ann Stat 1988; 16: $1141-1154$.

23 Fine JP, Gray RJ. A proportional hazards model for the subdistribution of a competing risk. J Am Stat Assoc 1999; 94: 496-509.

24 Eren S, Ciriş F. Diaphragmatic hernia: diagnostic approaches with review of the literature. Eur J Radiol 2005; 54: $448-459$. 
25 Kavic SM, Segan RD, George IM, et al. Classification of hiatal hernias using dynamic three-dimensional reconstruction. Surg Innov 2006; 13: 49-52.

26 Ginalski JM, Schnyder P, Moss AA, et al. Incidence and significance of a widened esophageal hiatus at CT scan. J Clin Gastroenterol 1984; 6: 467-470.

27 Vonk MC, van Die CE, Snoeren MM, et al. Oesophageal dilatation on high-resolution computed tomography scan of the lungs as a sign of scleroderma. Ann Rheum Dis 2008; 67: 1317-1321.

28 Lee S-W, Lien H-C, Chang C-S, et al. Impact of body mass index and gender on quality of life in patients with gastroesophageal reflux disease. World J Gastroenterol 2012; 18: 5090-5095.

29 Boeckxstaens GE. Alterations confined to the gastro-oesophageal junction: the relationship between low LOSP, TLOSRs, hiatus hernia and acid pocket. Best Pract Res Clin Gastroenterol 2010; 24: 821-829.

30 Caskey CI, Zerhouni EA, Fishman EK, et al. Aging of the diaphragm: a CT study. Radiology 1989; 171: 385-389.

31 Fein M, Ritter MP, DeMeester TR, et al. Role of the lower esophageal sphincter and hiatal hernia in the pathogenesis of gastroesophageal reflux disease. J Gastrointest Surg 1999; 3: 405-410.

32 Savarino E, Bazzica M, Zentilin P, et al. Gastroesophageal reflux and pulmonary fibrosis in scleroderma: a study using pH-impedance monitoring. Am J Respir Crit Care Med 2009; 179: 408-413.

33 Savarino E, Carbone R, Marabotto E, et al. Gastro-oesophageal reflux and gastric aspiration in idiopathic pulmonary fibrosis patients. Eur Respir J 2013; 42: 1322-1331. 